\title{
Comparison of Thematic Maps Using Symbolic Entropy
}

\author{
Manuel Ruiz Marín ${ }^{(1)}$, Fernando A. López Hernández ${ }^{(1)}$, Antonio Páez ${ }^{(2)}$ \\ (1) Technical University of Cartagena; (2) McMaster University
}

\begin{abstract}
Comparison of thematic maps is an important task in a number of disciplines. Map comparison has traditionally been conducted using cell-by-cell agreement indicators, such as the Kappa measure. More recently, other methods have been proposed that take into account not only spatially coincident cells in two maps, but also their surroundings or the spatial structure of their differences. The objective of this paper is to propose a framework for map comparison that considers 1) the patterns of spatial association in two maps, in other words, the map elements in their surroundings; 2) the equivalence of those patterns; and 3) the independence of patterns between maps. Two new statistics for the spatial analysis of qualitative data are introduced. These statistics are based on the symbolic entropy of the maps, and function as measures of map compositional equivalence and independence. As well, all inferential elements to conduct hypothesis testing are developed. The framework is illustrated using real and synthetic maps.
\end{abstract}

Key word: Thematic maps, map comparison, qualitative variables, spatial association, symbolic entropy, hypothesis tests 


\section{Introduction}

It is frequently the case when comparing maps that a researcher is interested in determining if two maps are significantly different, and if so, whether observed differences are random. There are numerous reasons why this type of information can be valuable. A number of distinct objectives have been identified in the literature (Boots and Csillag 2006; Kuhnert et al 2005; Stehman 1999). A researcher may be interested in accuracy assessment, that is, in assessing the degree of (mis)match between a reference map and one or more alternatives. For example, the maps could be of the same region but due to different producers, and end users need to understand the nature of the differences between maps (e.g., Wulder et al 2004). Alternatively, maps can be compared as part of a model validation exercise, when the comparison is made between a base map and the output of different modeling techniques or model specifications (e.g. discriminant analysis and neural networks; Foody 2004). In other situations, the comparison follows an interest to detect change, in order to determine whether the situation depicted in two maps of the same region remains unchanged (e.g., Mas 1999). Last but not least, there is a landscape ecology tradition that considers differences within or between regions (e.g., Gustafson 1998).

A number of different approaches have been proposed in the literature to compare maps, including examination of the frequency of classes in each map, the use of coincidence matrices, the kappa measure of agreement, fuzzy kappa, and other approaches (e.g., Foody 2002; Hagen 2003; Remmel 2009). Our objective in this paper is to propose a novel framework for map comparison that is based on the use of symbolic entropy to detect spatial patterns in thematic maps (Ruiz et al 2010). The framework follows a number of logically consistent steps. First, the maps are separately tested for spatial association of the qualitative variable to determine if a spatial structure is present or contrariwise, whether the values of the variable are spatially random. Secondly, if spatial patterns are detected, which would indicate a systematic process in the generation of the maps, then the compositional equivalence of the maps is assessed. Finally, the maps are tested to determine if they are independent, or rather a transition rule could exist that links them. The two questions that we pose with regards to the comparison of maps are: 
1) Are the maps significantly different? And if so:

2) Are the two maps independent?

Our procedure makes use of the $Q(m)$ statistic of spatial association introduced by Ruiz et al. (2010) for the analysis of mapped qualitative data. In addition, we introduce two new statistics needed for our comparison framework. The first of these new statistics is designed with the purpose of comparing the overall correspondence (i.e. the compositional equivalence) of map patterns. In broad terms, this is similar to the quantity agreement criterion, however considering series of spatially embedded map elements (i.e. map segments), as opposed to pairs of individual map elements. The second statistic is designed with the purpose of assessing whether two maps are independent.

We illustrate the application of the framework and all test statistics using real data collected from the Canada Land Inventory, and synthetic data. Various cases that can be found in practice are discussed. In the conclusions, we identify directions for future research.

\section{Background}

Two important aspects to consider when conducting map comparisons are the compositional and configurational characteristics of the maps. The distinction between these two is perhaps best captured by Pontius et al. (2004) as the quantity agreement and the location agreement between maps. A rough measure of compositional agreement is given by the total number of elements in a map that belong to class $i$ versus the number of elements in a second map that belong to the same class. In this way, two maps could have different composition if the coverage by class in one map is $70 \%$ of the surface while in the other is only $50 \%$. Of course, even if the proportion of cover by a specific class remained constant, it may have shifted over space, in which case the location agreement between the two maps would be poor. Coincidence matrices are used to record the degree of agreement between classes, and are the "golden standard” for accuracy assessment (Remmel 2009). Likewise, the kappa statistic, widely used in remote sensing applications, is based on an element-by-element comparison between the two maps. The comparison gives the number of spatially coincident cells in the maps that belong to the same or a different class. This approach introduces a measure of location agreement with respect to unchanged elements. Alternatives have 
been developed that improve the sensitivity of coincidence matrices and kappa to location and configuration differences (Pontius et al 2004; Remmel 2009).

Overall, there has been over the past few years an increased recognition that the spatial structure of maps is important in many comparative situations, and this is reflected in some recent and not-so-recent developments in the literature . An early example where the spatial structure of maps was identified as an important element in map comparison is a paper by Congalton (1988), where a difference image was generated based on remotely sensed datasets that classified pixels in a pair of maps as identical $(=0)$ or different (=1). The join-count statistic (Cliff and Ord 1981; Dacey 1968) was then applied to the resulting image to discover that the differences were not spatially random, but rather followed a systematic distribution suggestive of underlying topographical factors or systematic data processing error. A similar approach was used by Wulder et al. (2004) to detect whether maps obtained from different producers are different in systematic ways. There, two maps were simplified by collapsing all classes into forest and non-forest, and difference images were obtained. Two scenarios considered the differences between forest in the first map and non-forest in the second, and vice-versa. The results were helpful to identify spatial clusters (within a given level of statistical significance) where differences were common.

The method used by Congalton and Wulder et al. is based on the pair-wise comparison of map elements. Other approaches have been proposed that compare map segments instead, by embedding a map element as part of a neighborhood, notably the fuzzy kappa index (Hagen 2003; Hagen-Zanker et al 2005; Hagen-Zanker 2006) and moving window approaches (Kuhnert et al 2005). The fuzzy kappa compares spatially coincident map segments according to their degree of similarity. The innovation in the case of this method is the use of fuzzy concepts, so that instead of a crisp all-or-nothing decision rule for coding differences, two maps segments can be perfectly, somewhat, or not at all different. Recently Hagen-Zanker (2009) has modified the fuzzy kappa statistic to account for the effect of spatial autocorrelation on expected agreement. Kuhnert et al. (2005) describe a moving windows approach for calculating similarity indicators. According to this approach, a difference image is scanned by means of a moving window that registers the degree of dissimilarity for a map segment - if differences are coded as 1 and agreements as 0 and there is perfect coincidence within the window, the index would be zero. On the other hand, if the map segments are 
completely different at the location, the index would be one. The index is aggregated to produce a summary measure for the difference image.

Our approach shares some features with the fuzzy kappa and the moving windows approaches, in particular the way map elements are spatially embedded. In the case of Hagen (2003) the embedding provides the rational for the use of fuzzy concepts. The moving windows approach in contrast has much in common with various local statistics in spatial analysis (Fotheringham and Brunsdon 1999) despite the fact that in the end it is aggregated to produce a unique indicator for a map. In our case, the embedding is dictated by the mathematical framework of symbolic entropy. The fuzzy kappa is meant to be used as an interactive and exploratory tool to inform subjective interpretation of maps (Hagen-Zanker et al 2005, p.784; Hagen-Zanker 2009, p. 71). Our framework, in contrast, is staunchly inferential, and we derive all elements needed for conducting hypothesis testing.

With regards to statistical significance in the comparison of thematic maps, both Foody (2004) and Boots and Csillag (2006) propound the importance of conducting significance testing. On the other hand, Pontius et al. (2004) caution against the temptation of conducting hypothesis tests. Of the reasons advanced by Pontius et al., we agree that statistically significant differences should not be confused with practically important differences. We expect that this point will be fairly evident to most potential users of our approach. Finally, our approach explicitly incorporates spatial association considerations, and thus directly addresses an important characteristic of maps that is in need of attention (Hagen-Zanker 2009).

\section{A Framework for Map Comparisons}

Comparison of maps in our framework is done according to a sequence of logically consistent steps. The technical details of each step will be elaborated in subsequent sections of the paper. To understand the basic concepts of the framework, consider the maps shown in Figure 1. We will illustrate the procedure in reference to these maps. 

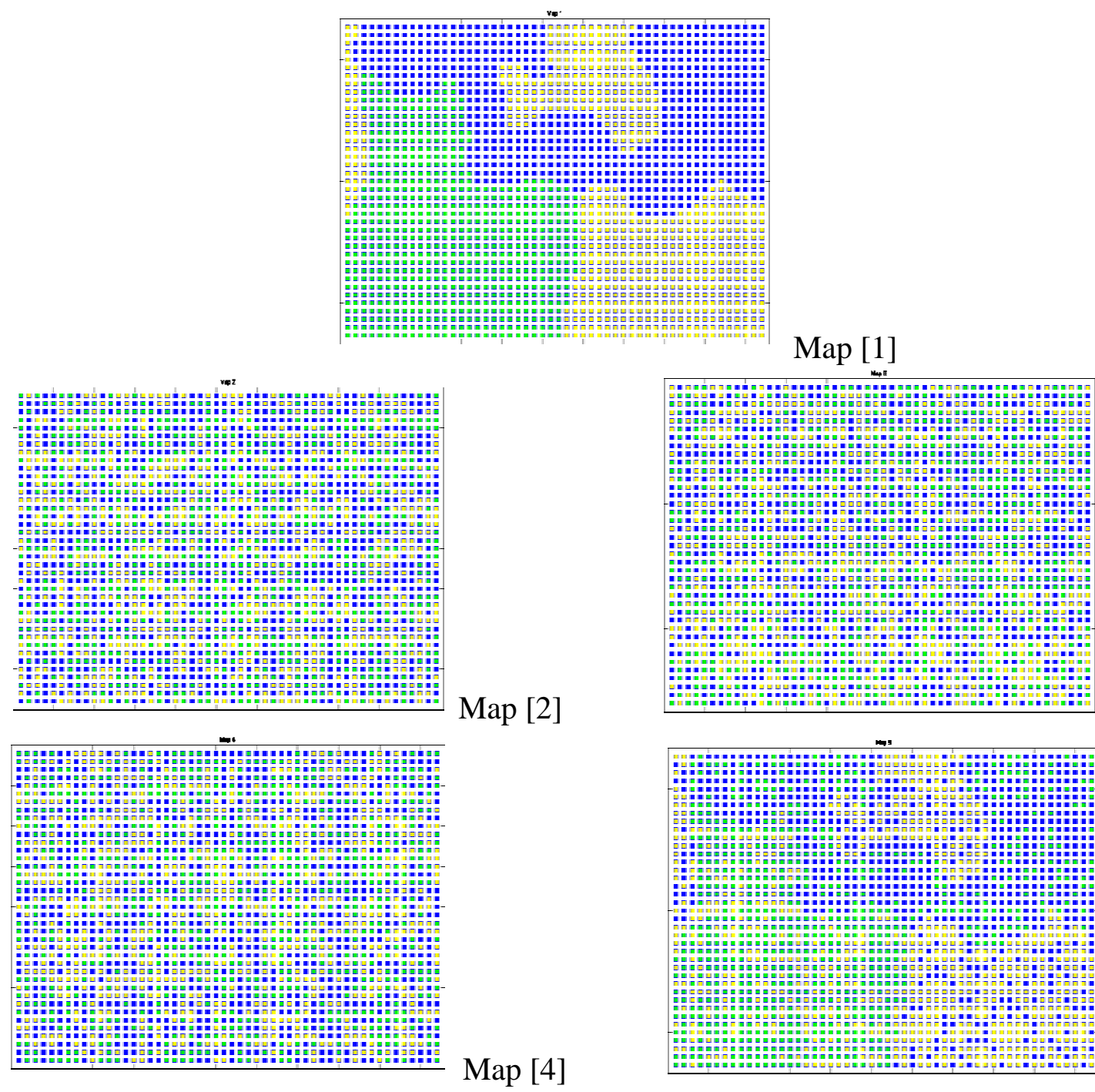

Map [3]
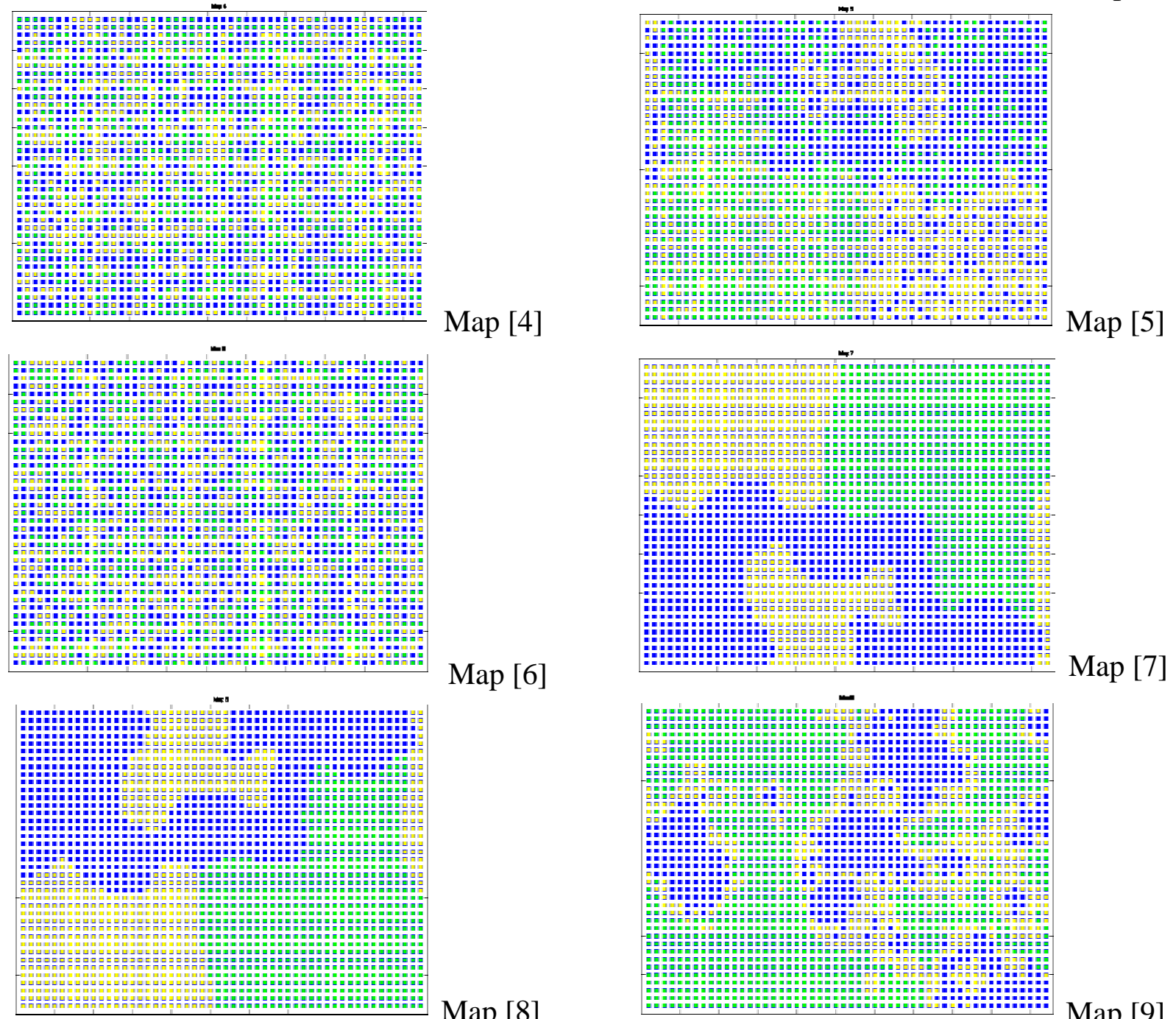

Map $[6]$

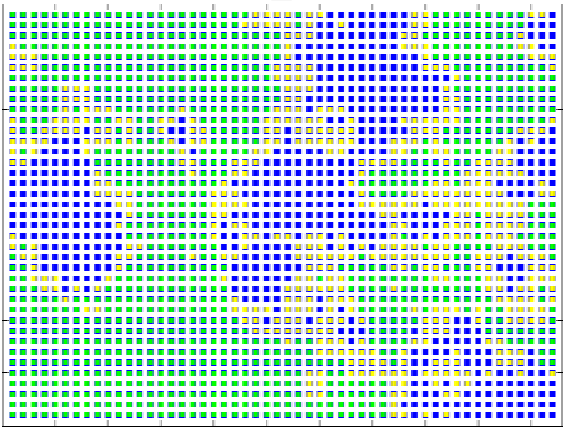

Figure 1. Maps in example. 
Each map in Figure 1 is composed of three different colors (classes) in various spatial arrangements. We take Map [1] as the base map. This map was extracted from the Canada Land Inventory, and depicts land uses in a region in the province of Ontario. The specific land uses are not relevant for our discussion here, and the methods described below can be applied to any thematic map. In addition to Map [1], we also generate a number of synthetic maps as follows. First, we generate three random maps that lack an ordered spatial pattern: Map [2] is generated using a random sequence of values drawn from the standard normal distribution and subsequently discretized; Map [3] is derived from Map [2] using a simple rule whereby each map class changes to one other color - specifically, all 1's become 2's, all 2's become 3's, and all 3's turn into 1's - the map is random, but not independent from Map [2]; Map [4] is another completely random distribution of values, unrelated to Maps [2] and [3]. Map [6] is derived from the base map based on random transitions, so that a cell can, with equal probability, stay in its current state, or change to one of the other two classes In addition, to the base map and a series of random maps, we also include a set of patterned maps: Map [5] is derived from Map [1] using structured transitions, with class 1 having a $30 \%$ chance of transitioning to 2, class 2 having a $10 \%$ of transitioning to 3 , and 3 having a 30\% chance of transitioning to class 2; Map [7] is the same as Map [1], but rotated 180 degrees; Map [8] is the mirror image of Map [1]; and finally Map [9] is simulated based on a pure autocorrelation model to produce a map pattern - this map has a high degree of spatial structure, but the data generating process is separate from any of the other patterned maps in the example. The relationships between the maps are shown in Figure 2 below. 


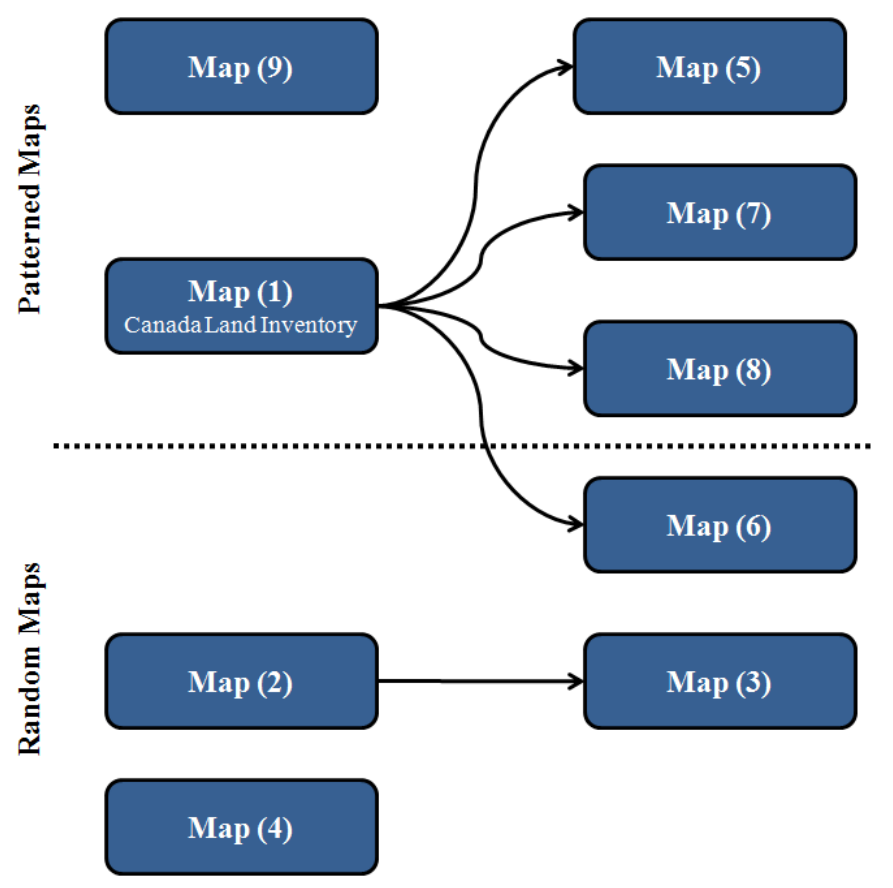

Figure 2. Relationships between maps in example.

Broadly speaking, a map can display a spatial pattern of association, or in other words, a spatially coherent distribution of values of the qualitative variable. The alternative is that the distribution of values is spatially random. Accordingly, we can identify the following cases for pair-wise comparison of maps:

Case 1. If one of the maps is spatially associated (i.e. there is spatial structure) and the other is random, it can be concluded that the maps are different and independent, since no organized transition rule can be used to convert one map into the other (i.e. Maps [1], [5], [7], [8], [9] : Maps [2], [3], [4], [6]).

The presence of spatial association in two maps is not by itself indicative of map agreement. When two maps are non-random, it becomes necessary to assess the overall similarity of the maps, that is, their compositional equivalence. If the overall composition of the maps is significantly different, the maps are of necessity different, and the question is whether the maps are independent, or a transition rule could possibly exist between them. If the overall composition of the maps is not significantly different, this would indicate compositional agreement, but not necessarily configurational agreement, and likewise it would be necessary to investigate whether the two maps are independent. This leads to the following cases:

Case 2. If map 1 and map 2 are each spatially associated, two issues need to be resolved. The first question is whether there is compositional agreement between the two maps. 
Case 2.1. The composition of the maps agrees, and therefore the maps could either be identical in all respects or identical in composition but not in configuration (Map [1] : Map [7] : Map [8]). Determine whether the maps are independent or a transition rule could exist.

Case 2.2. The composition of the maps does not agree, and therefore it can be concluded that the maps are different (Map [1] : Map [5] : Map [9]). Determine whether the maps are independent or a transition rule could exist.

A third case is of considerably less practical interest, but we mention it for completeness. This is when the two maps are spatially random (e.g., Map [2] : Map [3] : Map [4] : Map [6]). The element-wise differences could be random or not. It is straightforward to verify whether the transitions are random by looking at the frequency distribution of classes.

\section{Test statistics}

In this section we introduce the technical tools needed to implement our map comparison framework. We begin by defining some important terms (a number of these concepts are described in detail in Ruiz et al 2010). We will consider a map to be the representation of a discrete spatial process $\left\{X_{s}\right\}_{s \in S}$, where $S$ is a set of coordinates. The spatial process can take a number of discrete values or classes $A=\left\{a_{1}, a_{2}, \ldots, a_{k}\right\}$ that could be for example $k$ different land use classifications, types of forest cover, etc. The process can be considered to be spatially embedded, if we define a string that contains the classes found in a neighborhood of size $m$, called an $m$-surrounding. The $m$ surrounding is similar to the neighborhood vector $N$ used in the fuzzy kappa measure of agreement (Hagen 2003; Hagen-Zanker et al 2005):

$$
X_{s_{0}}(m)=\left(X_{s_{0}}, X_{s_{1}}, \ldots, X_{s_{m-1}}\right)
$$

The string is defined for a specific coordinate corresponding to a location in the map ( $s_{0} \in S$ ) where $s_{1}, s_{2}, \ldots, S_{m-1}$ are the $m-1$ nearest neighbors to $s_{0}$. In other words, $X_{s_{0}}(m)$ is a list of values that represents a map segment of size $m$ centered on location $s_{0}$. The values in the string are the classes found in a neighborhood of size $m$. We will denote the set of the $m-1$-nearest neighbors by $N_{s}=\left\{s_{1}, s_{2}, \ldots, s_{m-1}\right\}$. As an example, 
consider the simple case of a lineal transect with $k=2$ ( $a_{1}=$ White $=0$, and $a_{2}=$ Blue $=1$; see Figure 3).

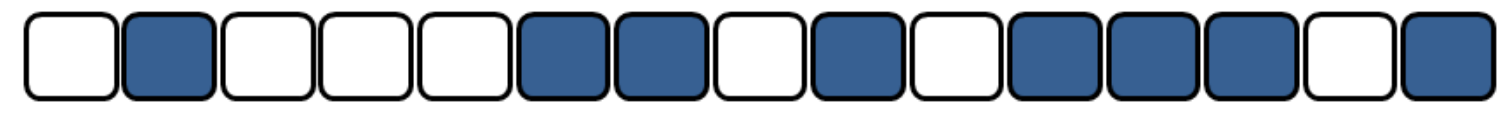

Figure 3. Lineal transect with $k=2$.

If the size of the $m$-surrounding is selected as 3 , then $X(3)$ for the second cell from the left is $\{$ Blue, White, White or equivalently $\{1,0,0\} . X(3)$ for the fifth cell from the left is $\{$ White, Blue, White $\}$ or $\{0,1,0\}$.

It is easy to see that since the number of different classes in the map is $k$, the number of all possible values (the combination of classes) that an $m$-surrounding $M_{s}(m)$ can take is $k^{m}$. In the example above, with $k=2$ and $m=3$, there are eight possible combinations of classes (see Table 1). Each unique combination of classes is called a symbol, which we denote by $\sigma_{i}\left(\mathrm{i}=1,2, \ldots, k^{m}\right)$

Table 1: List of symbols for $k=2, m=3$.

\begin{tabular}{|l|l|l|l|}
\hline$\sigma_{1}=$ & $\{0,0,0\}$ & $\sigma_{5}=$ & $\{1,1,0\}$ \\
\hline$\sigma_{2}=$ & $\{1,0,0\}$ & $\sigma_{6}=$ & $\{1,0,1\}$ \\
\hline$\sigma_{3}=$ & $\{0,1,0\}$ & $\sigma_{7}=$ & $\{0,1,1\}$ \\
\hline$\sigma_{4}=$ & $\{0,0,1\}$ & $\sigma_{8}=$ & $\{1,1,1\}$ \\
\hline
\end{tabular}

Let:

$\Gamma=\left\{\sigma_{1}, \sigma_{2}, \ldots, \sigma_{k^{m}}\right\}$

be the set of all of these possible values. For a given map $\left\{X_{s}\right\}_{s \in S}$ we say that a location $s$ is of $\sigma_{i}$-type if and only if $X_{s}(m)=\sigma_{i}$. In the example, the second cell from the left is said to be of $\sigma_{2}$-type, and the fifth cell from the left is of $\sigma_{3}$-type. Each cell can be assigned a unique symbol, out of the finite list of possible symbols. The cells on the edges would use the two nearest neighbors, and would therefore be of type $\sigma_{3}$ (on the left) and of type $\sigma_{6}$ (on the right). Precise rules for selecting the neighbors are described in more detail below.

Let $\sigma_{i} \in \Gamma$ and denote by:

$n_{\sigma_{i}}=\sharp\left\{s \in S \mid X_{s}(m)=\sigma_{i}\right\}$, 
the size of the subset of $S$ formed by all the elements of $\sigma_{i}$-type. Given this, it is easy to compute the empirical relative frequency of a symbol $\sigma_{i} \in \Gamma$ as:

$p\left(\sigma_{i}\right):=p_{\sigma_{i}}=\frac{\sharp\left\{s \in S \mid s \text { is of } \sigma_{i}-\text { type }\right\}}{|S|}$

where by $|S|$ we denote the cardinality of the set $S$ (the number of symbolized locations).

Now, under this setting, we can define the symbolic entropy of a thematic map $\left\{X_{s}\right\}_{s \in S}$ for an embedding dimension $m \geq 2$. This entropy is defined as the Shanon's entropy of the $k^{m}$ distinct symbols as follows:

$h_{X}(m)=-\sum_{i=1}^{k^{m}} p_{\sigma_{i}} \ln \left(p_{\sigma_{i}}\right)$

Symbolic entropy, $h(m)$, is the information contained in comparing the $m$ surroundings in the map. Note that $0 \leq h(m) \leq \ln \left(k^{m}\right)$, where the lower bound is attained when only one symbol occurs, and the upper bound is for a completely random system (i.i.d. spatial sequence) where all $k^{m}$ possible symbols appear with the same probability.

In this section we introduce the tests that allow the researcher to assess the degree of spatial association (structure) of a thematic map, and compare two maps in terms of (1) their compositional equivalence and (2) their independence. In order to do so, we will consider two maps $\left\{X_{s}\right\}_{s \in S}$ and $\left\{Y_{s}\right\}_{s \in S}$ with the same zoning system, and denote the symbolic frequencies in each map, and their combination, by (the statistics can be expanded to consider more than two maps simultaneously):

$$
\begin{array}{cc}
n_{\sigma_{i}}=\sharp\left\{s \in S \mid X_{s}(m)=\sigma_{i}\right\} ; & p_{\sigma_{i}}=\frac{n_{\sigma_{i}}}{|S|} \\
m_{\sigma_{i}}=\sharp\left\{s \in S \mid Y_{s}(m)=\sigma_{i}\right\} ; & q_{\sigma_{i}}=\frac{m_{\sigma_{i}}}{|S|}, \\
c_{\sigma_{i}}=n_{\sigma_{i}}+m_{\sigma_{i}} ; & g_{\sigma_{i}}=\frac{c_{\sigma_{i}}}{2|S|}
\end{array}
$$

\subsection{Spatial structure: The $Q(m)$ statistic}

Testing for spatial structure (i.e. spatial association) of a qualitative variable based on symbolic entropy is discussed in Ruiz et al. (2010). In this section, we limit ourselves to 
reviewing some key concepts of the relevant statistic. The reader is directed to the reference for further information. The $Q(m)$ statistic is defined as follows:

$$
Q(m)=-2|S|\left[\sum_{i=1}^{k^{m}} \frac{n_{\sigma_{i}}}{|S|} \sum_{j=1}^{k} \alpha_{i j} \ln \left(q_{j}\right)+h(m)\right]
$$

where $\alpha_{i j}$ the number of times that class $a_{j}$ appears in symbol $\sigma_{i}$ and $q_{j}=P\left(X=a_{j}\right)$. The statistic is essentially a likelihood ratio test that compares the symbolic entropy of the map to the expected symbolic entropy under the null hypothesis of spatial independence (i.e. where all symbols appear with similar probability contingent on the frequency of classes). The statistic can be shown to be $\chi^{2}$ distributed with $k^{m}-1$ degrees of freedom (Ruiz et al 2010), and the decision rule at a 100(1- $\alpha) \%$ confidence level is as follows:

Reject null hypothesis if $Q(m)>\chi_{\alpha}^{2}$ Otherwise do not reject the null hypothesis

In order to implement the test, the researcher must make a number of decisions. First, the researcher must decide the size of $m$ for the $m$-surrounding; there is a certain degree of flexibility in doing so, however limited by some statistical and interpretive considerations. In order to achieve a sufficient approximation of the $\chi^{2}$ distribution, it is strongly advisable to work with at least $5 k^{m}$ symbolized observations (Rohatgi 1976, chapter 10); in other words, there must be at least five times as many symbolized observations as symbols exist. Thus, the size of $m$ may be constrained by the size of the map, with larger surroundings only practicable in large maps. In terms of interpretation, a large number of symbols capturing a high level of specificity may also be difficult to interpret. On the other hand, if spatial association is detected for a surrounding of size $m$, the statistic guarantees that the association exists for the full surrounding or even only part of it. The presence of spatial association at higher scales remains indeterminate.

Once a decision has been made regarding the size of the m-surrounding, a rule is needed to identify $m-1$ nearest neighbors for each location that will be symbolized. The rule implemented in Ruiz et al. (2010) is as follows:

(a) The distance of the $m-1$ neighbours from $s_{0}$ satisfies the condition that $\rho_{1}^{0} \leq \rho_{2}^{0} \leq \ldots \leq \rho_{m-1}^{0}$; and 
(b) In the case of a tie in terms of the distance from $s_{0}$, (i.e. if $\rho_{i}^{0}=\rho_{i+1}^{0}$ ) then precedence goes to the smaller angle (i.e. $\theta_{i}^{0}<\theta_{i+1}^{0}$ ).

Plainly, neighbors are selected in order of proximity, and if there are ties according to this criterion, selection proceeds by direction. This ensures the uniqueness of $X_{m}(s)$ for all $s \in S$. Other rules are certainly possible, for example by changing the criteria to select neighbors by direction if anisotropic processes are suspected; however, we do not pursue this idea further in the present paper.

\subsection{Compositional equivalence: The $Q_{E}$ test}

After testing the maps for spatial structure, if the two maps are found to be non-random, we are interested in testing whether the composition of the maps is equivalent. In other words, we now wish to ascertain whether symbols appear with similar frequencies in both maps. Hence, the null hypothesis that we want to test is whether the symbolic distributions for both maps are equivalent, or:

$$
H_{0}:\left\{X_{s}\right\}_{s \in S} \text { and }\left\{Y_{s}\right\}_{s \in S} \text { follow the same distribution. }
$$

The null hypothesis can be restated in terms of symbols as follows:

$$
H_{0}: p_{\eta_{i}}=q_{\eta_{i}} \text { for all } i=1,2, \ldots, k^{m}
$$

that is, the frequency of symbols is identical in the two maps, for all symbols. In order to develop a test statistics, we define, for a symbol $\sigma_{i} \in \Gamma$, the following random variables:

$$
Z_{\sigma_{i} s}(X)=\left\{\begin{array}{lc}
1 & \text { if } X_{m}(s)=\sigma_{i} \\
0 & \text { otherwise, }
\end{array} Z_{\sigma_{i} s}(Y)= \begin{cases}1 & \text { if } Y_{m}(s)=\sigma_{i} \\
0 & \text { otherwise }\end{cases}\right.
$$

Then $Z_{\eta_{i} s}(X)$ (respectively $Z_{\eta_{i} s}(Y)$ ) is a Bernoulli variable with probability of “success" $p_{\eta_{i}}$ (resp. $q_{\eta_{i}}$ ), where "success" means that $s$ is of $\sigma_{i}$-type.

Now assume that set $S$ is finite and of order $R$. Then we are interested in knowing how many $s$ 's are of $\sigma_{i}$-type for all symbol $\sigma_{i} \in S$ in each of the maps. In order to answer the question, we construct the following variables

$$
T_{\sigma_{i}}(X)=\sum_{s \in S} Z_{\sigma_{i} s(X)} \quad T_{\sigma_{i}}(Y)=\sum_{s \in S} Z_{\sigma_{i} s(Y)}
$$


The variable $T_{\sigma_{i}}$ can take the values $\{0,1,2, \ldots, R\}$. Notice that not all the variables $Z_{\sigma_{i} s}$ are independent (due to the overlapping of some $m$-surroundings), and therefore $T_{\sigma_{i}}$ is not exactly a Binomial random variable. Nevertheless, the sum of dependent indicators can be approximated to a Binomial random variable whenever the dependencies among the indicators are weak (Soon 1996).

A procedure to obtain a good approximation to a Binomial distribution is described in Ruiz et al. (Ruiz et al 2010). Briefly, we can consider as a set of locations the subset $\widetilde{S}$ formed by those coordinates in $S$ such that for any two coordinates $s, s^{\prime} \in \widetilde{S}$ the sets of nearest neighbors of $s$ and $s^{\prime}$ have a small (or even empty) intersection, that is:

$$
\left|N_{s} \cap N_{s^{\prime}}\right|=r
$$

for a small enough positive integer $r$. We will call the integer $r$ as the overlapping degree.

Now we propose a construction of the set $\widetilde{S}$. First chose a location $\tilde{S}_{0} \in S$ at random and fix an integer $r$ with $0 \leq r<m$. Let $N_{\tilde{s}_{0}}=\left\{s_{1}^{0}, s_{2}^{0}, \ldots, s_{m-1}^{0}\right\}$ be the set of nearest neighbors to $\tilde{S}_{0}$, where the $s_{i}^{0}$ 's are ordered by distance to $\tilde{s}_{0}$. Let us call $\tilde{s}_{1}=s_{m-r-1}^{0}$ and define $A_{0}=\left\{\tilde{S}_{0}, s_{1}^{0}, \ldots, s_{m-r-2}^{0}\right\}$. Take the set of nearest neighbors to $\tilde{S}_{1}$, namely $N_{\tilde{s}_{1}}=\left\{s_{1}^{1}, s_{2}^{1}, \ldots, s_{m-1}^{1}\right\}$, in the set of locations $S \backslash A_{0}$ and define $\tilde{s}_{2}=s_{m-r-1}^{1}$. Now for $i>1$ we define $\tilde{s}_{i}=s_{m-r-1}^{i-1}$ where $s_{m-r-1}^{i-1}$ is in the set of nearest neighbors to $\tilde{S}_{i-1}$, $N_{\tilde{s}_{i-1}}=\left\{s_{1}^{i-1}, s_{2}^{i-1}, \ldots, S_{m-1}^{i-1}\right\}$, in the set $S \backslash\left\{\cup_{j=0}^{i-1} A_{j}\right\}$. Continue this process while there are locations to be symbolized. Therefore we have constructed a set of locations:

$$
\widetilde{S}=\left\{\tilde{S}_{0}, \tilde{S}_{1}, \ldots, \tilde{S}_{M}\right\}
$$

such that the variables:

$$
T_{\sigma_{i}}(X)=\sum_{s \in \tilde{S}} Z_{\sigma_{i} s(X)}=B\left(|\widetilde{S}|, p_{\sigma_{i}}\right) \quad T_{\sigma_{i}}(Y)=\sum_{s \in \tilde{S}} Z_{\sigma_{i} s(Y)}=B\left(|\widetilde{S}|, q_{\sigma_{i}}\right)
$$

based on $\widetilde{S}$ can be approximated to a binomial distribution for a suitable choice of $r$ ( $r$ small enough) for all $i=1,2, \ldots, k^{m}$. Moreover, when $r=0$ (i.e. no overlap is allowed) we exactly have Binomial random variables. Note that the maximum number of locations that can be symbolized with an overlapping degree $r$ is $M=\left[\frac{R-m}{m-r}\right]+1$, where 
$[x]$ denotes the integer part of a real number $x$, and therefore reducing the degree of overlap also implies that the number of symbolized locations will be smaller than the number of observations in the sample.

Now, the distribution of the $2 k^{m}$ random variables:

$T=\left(T_{\sigma_{1}}(X), \ldots, T_{\sigma_{k^{m}}}(X), T_{\sigma_{1}}(Y), \ldots, T_{\sigma_{k^{m}}}(Y)\right)$

is a multinomial distribution and its likelihood function $L\left(p_{\sigma_{1}}, \ldots, p_{\sigma_{k^{m}}}, q_{\sigma_{1}}, \ldots, q_{\sigma_{k^{m}}}\right)$ is given by:

$\frac{2 M !}{n_{\sigma_{1}} ! \cdots n_{\sigma_{k^{m}}} ! m_{\sigma_{1}} ! \cdots m_{\sigma_{k^{m}}} !} p_{\sigma_{1}}^{n_{\sigma_{1}}} \cdots p_{\sigma_{k^{m}}}^{n_{\sigma^{m}}} q_{\sigma_{1}}^{m_{\sigma_{1}}} \cdots q_{\sigma_{k^{m}}}^{m_{\sigma^{m}}}$

In order to obtain the maximum likelihood estimators $\hat{p}_{\sigma_{i}}$ and $\hat{q}_{\sigma_{i}}$ of $p_{\sigma_{i}}$ and $q_{\sigma_{i}}$ respectively and taking into account that:

$\sum_{i=1}^{n} p_{\sigma_{i}}=1=\sum_{i=1}^{n} q_{\sigma_{i}}$

we solve the following system of equations:

$\frac{\partial \ln \left(L\left(p_{\sigma_{1}}, \ldots, p_{\sigma_{k^{m}}}, q_{\sigma_{1}}, \ldots, q_{\sigma_{k^{m}}}\right)\right)}{\partial p_{\sigma_{i}}}=0 \quad \frac{\partial \ln \left(L\left(p_{\sigma_{1}}, \ldots, p_{\sigma_{k^{m}}}, q_{\sigma_{1}}, \ldots, q_{\sigma_{k^{m}}}\right)\right)}{\partial q_{\sigma_{i}}}=0$

for all $i=1, \ldots, k^{m}-1$ to obtain:

$\hat{p}_{\sigma_{i}}=\frac{n_{\sigma_{i}}}{M} \quad \hat{q}_{\sigma_{i}}=\frac{m_{\sigma_{i}}}{M}$.

Denote by $p_{\sigma}^{(0)}$ and $q_{\sigma}^{(0)}$ the probabilities under the null for all symbols $\sigma \in \Gamma$ Then the likelihood ratio statistic is (see for example Lehmann 1986):

$\lambda(T)=\frac{p_{\sigma_{1}}^{(0) n_{\sigma_{1}}} \cdots p_{\sigma_{k^{m}}}^{(0) n_{\sigma^{m}}} q_{\sigma_{1}}^{(0) m_{\sigma_{1}}} \cdots q_{\sigma_{k^{m}}}^{(0) m_{\sigma^{m}}}}{p_{\sigma_{1}}^{n_{\sigma_{1}}} \cdots p_{\sigma_{k^{m}}}^{n_{\sigma_{k^{m}}}} q_{\sigma_{1}}^{m_{\sigma_{1}}} \cdots q_{\sigma_{k^{m}}}^{m_{\sigma^{m}}}}$

Now, under the null, we have that $p_{\sigma_{i}}=q_{\sigma_{i}}$ and hence $p_{\sigma_{i}}=\frac{1}{2} g_{\sigma_{i}}$ for all $i=1,2, \ldots, k^{m}$. Therefore: 


$$
\lambda(T)=\frac{\left(\frac{1}{2}\right)^{2 M} g_{\sigma_{1}}^{n_{\sigma_{1}}+m_{\sigma_{1}}} \cdots g_{\sigma_{k^{m}}}^{n_{\sigma^{m}}+m_{\sigma_{k^{m}}}}}{p_{\sigma_{1}}^{n_{\sigma_{1}}} \cdots p_{\sigma_{k^{m}}}^{n_{\sigma^{m}}} q_{\sigma_{1}}^{m_{\sigma_{1}}} \cdots q_{\sigma_{k^{m}}}^{m_{\sigma_{k^{m}}}}}
$$

We define the structural equivalence test as $Q_{E}(m)=-2 \ln (\lambda(T))$, which is known to asymptotically follow a $\chi^{2}$ distribution with $k^{m}-1$ degrees of freedom (Lehmann 1986). Hence, the estimator $\hat{Q}_{E}(m)$ of $Q_{E}(m)$ can be shown to be:

$$
\begin{aligned}
\hat{Q}_{E}(m) & =-2\left[2 M \ln \left(\frac{1}{2}\right)+\sum_{i=1}^{k^{m}} c_{\sigma_{i}} \ln \left(\frac{c_{\sigma_{i}}}{2 M}\right)-\sum_{i=1}^{k^{m}} n_{\sigma_{i}} \ln \left(\frac{n_{\sigma_{i}}}{M}\right)-\sum_{i=1}^{k^{m}} m_{\sigma_{i}} \ln \left(\frac{m_{\sigma_{i}}}{M}\right)\right] \\
& =-4 M\left[\ln \left(\frac{1}{2}\right)+\sum_{i=1}^{k^{m}} \frac{c_{\sigma_{i}}}{2 M} \ln \left(\frac{c_{\sigma_{i}}}{2 M}\right)-\sum_{i=1}^{k^{m}} \frac{n_{\sigma_{i}}}{2 M} \ln \left(\frac{n_{\sigma_{i}}}{M}\right)-\sum_{i=1}^{k^{m}} \frac{m_{\sigma_{i}}}{2 M} \ln \left(\frac{m_{\sigma_{i}}}{M}\right)\right] \\
& =4 M\left[\ln (2)+\hat{h}(m)-\frac{1}{2} \hat{h}_{X}(m)-\frac{1}{2} \hat{h}_{Y}(m)\right]
\end{aligned}
$$

where $h(m)=-\sum_{i=1}^{k^{m}} g_{\sigma_{i}} \ln \left(g_{\sigma_{i}}\right)$ is the total symbolic entropy. We have proved the following result.

Theorem 1. Let $\left\{X_{s}\right\}_{s \in \tilde{S}}$ and $\left\{Y_{s}\right\}_{s \in \tilde{S}}$ be two maps with $|\tilde{S}|=M$. Let $\Gamma=\left\{\sigma_{1}, \sigma_{2}, \ldots, \sigma_{k^{m}}\right\}$ be the set of $k^{m}$ symbols as defined in (2) If $\left\{X_{s}\right\}_{s \in \tilde{S}}$ and $\left\{Y_{s}\right\}_{s \in \tilde{S}}$ are structural equivalent then:

$$
\hat{Q}_{E}(m)=4 M\left[\ln (2)+\hat{h}(m)-\frac{1}{2} \hat{h}_{X}(m)-\frac{1}{2} \hat{h}_{Y}(m)\right]
$$

is asymptotically $\chi_{k^{m}-1}^{2}$ distributed.

The $Q_{E}(m)$-test can be generalized to the case of $N$ maps, $\left\{X_{1 s}\right\}_{s \in \tilde{S}},\left\{X_{2 s}\right\}_{s \in \tilde{S}}, \ldots,\left\{X_{N s}\right\}_{s \in \tilde{S}}$ as it can be seen in the next corollary. The proof is straightforward following the steps of the proof of Theorem 1.

Corollary 2. Let $\left\{X_{i s}\right\}_{s \in \tilde{S}} i=1,2 \ldots, N$ be $N$ maps with $|\widetilde{S}|=M$. Let $\Gamma=\left\{\sigma_{1}, \sigma_{2}, \ldots, \sigma_{k^{m}}\right\}$ be the set of $k^{m}$ symbols as defined in (2) If $\left\{X_{i s}\right\}_{s \in \tilde{S}}$ are structural equivalent for all $i=1,2, \ldots, N$ then

$$
\hat{Q}_{E}(m)=2 N M\left[\ln (N)+\hat{h}(m)-\frac{1}{N} \sum_{i=1}^{N} \hat{h}_{X_{i}}(m)\right]
$$


is asymptotically $\chi_{(N-1)\left(k^{m}-1\right)}^{2}$ distributed.

\subsection{Map independence: The $Q_{I}$ test}

Two maps known to have spatial association as determined by $Q(m)$ are organized and therefore in principle interesting. The maps can be compositionally equivalent as determined by the $Q_{E}(m)$ statistic. This does not yet indicate whether the maps are related in a non-random fashion. We are therefore interested in assessing whether the maps, namely $\left\{X_{s}\right\}_{s \in S}$ and $\left\{Y_{s}\right\}_{s \in S}$, are dependent or independent maps. In this case, independence means that a non-random transformation rule does not exist that could be used to convert one map into the other. Note that two independent maps can be structurally equivalent and viceversa. Then next step is to test for independence. First we are going to introduce some definitions and notation that will be needed in order to construct the statistic.

Consider the map overlay $\left\{W_{s}=\left(X_{s}, Y_{s}\right)\right\}_{s \in S}$. Let $\Omega=\Gamma \times \Gamma$ be the direct product of the set of symbols $\Gamma=\left\{\sigma_{1}, \sigma_{2}, \ldots, \sigma_{k^{m}}\right\}$. We will call the elements in $\Omega$ symbols for the 2dimensional map. Next we define the $m$-surrounding associated to the 2-dimensional $\operatorname{map}\left\{W_{s}=\left(X_{s}, Y_{s}\right)\right\}_{s \in S}$ as $W_{s}(m)=\left(X_{s}(m), Y_{s}(m)\right)$. Now given a symbol $\left(\sigma_{i}^{x}, \sigma_{j}^{y}\right) \in \Omega$ we will say that a location $s$ is of $\left(\sigma_{i}^{x}, \sigma_{j}^{y}\right)$-type for $W$ if and only if the location $s$ is of $\sigma_{i}^{x}$-type for the map $X$ and of $\sigma_{j}^{y}$-type for the map $Y$. Let $d_{i j}=\sharp\left\{s \in S \mid W_{s}(m)=\left(\sigma_{i}, \sigma_{j}\right)\right\}$ and denote by $p_{i j}=\frac{d_{i j}}{|S|}$ the probability of the symbol $\left(\sigma_{i}^{x}, \sigma_{j}^{y}\right)$ to occur. Then we can define the symbolic entropy of the map overlay as $h_{W}(m)=-\sum_{i=1}^{k^{m}} \sum_{j=1}^{k^{m}} p_{i j} \ln \left(p_{i j}\right)$.

Define the indicator function $I_{i j s}=\left\{\begin{array}{cc}1 & \text { if } s \text { is of }\left(\sigma_{i}^{x}, \sigma_{j}^{y}\right) \text {-type for } \mathrm{W} \\ 0 & \text { otherwise }\end{array}\right.$

Hence we have that $I_{i j s}=B\left(p_{i j}\right)$ is a Bernoulli random variable with probability of "success" $p_{i j}$. As in the previous section we can define a subset of 
locations $\widetilde{S}$ such that $\sum_{s \in \mathcal{S}} I_{i j s}$ can be approximated to a binomial distribution for a suitable choice of $r$ ( $r$ small enough $)$ for all $i, j=1,2, \ldots, k^{m}$. Therefore $F_{i j}=\sum_{s \in \mathcal{S}} I_{i j s}=B\left(M, p_{i j}\right)$, is a binomial random variable where $M$ denotes the cardinality of the set $\widetilde{S}$. Then:

$F=\left(F_{12}, F_{13}, \ldots, F_{k k-1}\right)$

is a multinomial distribution. Now we are interested in testing for the following null:

$H_{0}$ : The maps $\left\{\mathrm{X}_{\mathrm{s}}\right\}_{s \in S}$ and $\left\{Y_{\mathrm{s}}\right\}_{s \in S}$ are independent.

This null hypothesis can be restated in terms of symbols as:

$H_{0}: \quad p_{i j}=p_{\sigma_{i}} q_{\sigma_{j}}$

Following the same steps as in the previous section we get that under the null the likelihood ratio statistic remains as:

$$
\lambda(F)=\frac{\prod_{i=1}^{k} \prod_{j=1}^{k} p_{\sigma_{i}}^{d_{i j}} q_{\sigma_{j}}^{d_{i j}}}{\prod_{i \neq j} p_{i j}^{d_{i j}}}
$$

We then define $Q_{I}(m)=-2 \ln (\lambda(F))$, which asymptotically follows a $\chi^{2}$ distribution with $k^{m}\left(k^{m}-2\right)+1$ degrees of freedom see (see Lehmann 1986). Hence, and taking into account that $\sum_{j=1}^{k^{m}} d_{i j}=d_{i \bullet}=n_{\sigma_{i}}$ and $\sum_{i=1}^{k^{m}} d_{i j}=d_{\bullet j}=m_{\sigma_{j}}$ and that $p_{\sigma_{i}} q_{\sigma_{j}}=\frac{n_{\sigma_{i}}}{M} \frac{m_{\sigma_{j}}}{M}$ we obtain that the estimator $\hat{Q}_{I}(m)$ of $Q_{I}(m)$ is:

$$
\begin{aligned}
\hat{Q}_{I}(m) & =-2\left[\sum_{i=1}^{k^{m}} d_{i \cdot} \ln \left(\frac{n_{\sigma_{i}}}{M}\right)+\sum_{i=1}^{k^{m}} d_{\cdot j} \ln \left(\frac{m_{\sigma_{j}}}{M}\right)-\sum_{i=1}^{k^{m}} \sum_{j=1}^{k^{m}} d_{i j} \ln \left(\frac{d_{i j}}{M}\right)\right] \\
& =2 M\left[-\sum_{i=1}^{k^{m}} \frac{d_{i \cdot}}{M} \ln \left(\frac{n_{\sigma_{i}}}{M}\right)-\sum_{i=1}^{k^{m}} \frac{d_{\cdot j}}{M} \ln \left(\frac{m_{\sigma_{j}}}{M}\right)-\sum_{i=1}^{k^{m}} \sum_{j=1}^{k^{m}} \frac{d_{i j}}{M} \ln \left(\frac{d_{i j}}{M}\right)\right] \\
& =2 M\left[\hat{h}_{X}(m)+\hat{h}_{X}(m)-\hat{h}_{W}(m)\right] .
\end{aligned}
$$

Therefore we have proved the following result. 
Theorem 3. Let $\left\{X_{s}\right\}_{s \in \tilde{S}}$ and $\left\{Y_{s}\right\}_{s \in \tilde{S}}$ be two maps and let $\left\{W_{s}=\left(X_{1 s}, X_{2 s}\right)\right\}_{s \in \tilde{S}}$ be a 2-dimensional map with $|\widetilde{S}|=M$. Let $\Gamma=\left\{\sigma_{1}, \sigma_{2}, \ldots, \sigma_{k^{m}}\right\}$ be the set of $k^{m}$ symbols as defined in (2). If $\left\{X_{s}\right\}_{s \in \tilde{S}}$ and $\left\{Y_{s}\right\}_{s \in \tilde{S}}$ are independent then

$Q_{I}(m)=2 M\left[h_{X}(m)+h_{Y}(m)-h_{W}(m)\right]$

is asymptotically $\chi_{k^{m}\left(k^{m}-2\right)+1}^{2}$ distributed.

The $Q_{I}(m)$-test can be generalized to the case of $N$ maps, $\left\{X_{1 s}\right\}_{s \in \tilde{S}},\left\{X_{2 s}\right\}_{s \in \tilde{S}}, \ldots,\left\{X_{N s}\right\}_{s \in \tilde{S}}$ as it can be seen in the next corollary. The proof is straightforward following the steps of the proof of Theorem 3.

Corollary 4. Let $\left\{X_{i s}\right\}_{s \in \tilde{S}} \quad i=1,2 \ldots, N \quad$ be $N$ maps and let $\left\{W_{s}=\left(X_{1 s}, X_{2 s}, \ldots, X_{N s}\right)\right\}_{s \in \tilde{S}}$ be a N-dimensional map with $|\widetilde{S}|=M$. Let $\Gamma=\left\{\sigma_{1}, \sigma_{2}, \ldots, \sigma_{k^{m}}\right\}$ be the set of $k^{m}$ symbols as defined in (2) If $\left\{X_{i s}\right\}_{s \in \tilde{S}}$ are independent for all $i=1,2, \ldots, N$ then

$Q_{I}(m)=2 M\left[\sum_{i=1}^{N} h_{X_{i}}(m)-h_{W}(m)\right]$

is asymptotically $\chi_{k^{N m}-N\left(k^{m}-1\right)-1}^{2}$ distributed.

\section{Illustration}

The framework proposed in the preceding sections is illustrated with reference to the maps in Figure 1. The first step is to test the maps for spatial association. We calculate the $Q(m)$ statistic for all maps using an $m$-surrounding of 4 and symbolize the observations using an overlap degree $r=1$. The results of applying the statistic to the maps appear in Table 2. The table shows the value of the statistic for each map, the probability value, and the decision (at a 95\% level of confidence). In addition, we also include a figure showing the frequency distribution of equivalent symbols in each map. The equivalent symbols are a reduced form of the symbols that display only the number of cases of each class in an $m$-surrounding, instead of all proximity information; this reduction of information facilitates interpretation. The figures are illustrative only, and the tests are based on the standard symbolization. The vertical axis in the figure is the proportion of the total, and in the horizontal axis the equivalent symbols appear. For 
example $\left\{\begin{array}{lll}0 & 0 & 4\end{array}\right\}$ is the symbol indicating an $m$-surrounding of 4 where all map elements are of class $a_{3} ;\left\{\begin{array}{lll}2 & 1 & 1\end{array}\right\}$ is the symbol indicating an $m$-surrounding of 4 where two elements are of class $a_{1}$, one is of class $a_{2}$, and one is of class $a_{3}$.

Table 2: $Q(m)$ test for spatial association (non-random maps in bold, $p$-values in parentheses). Test is conducted for $m=4$ and $r=1$.

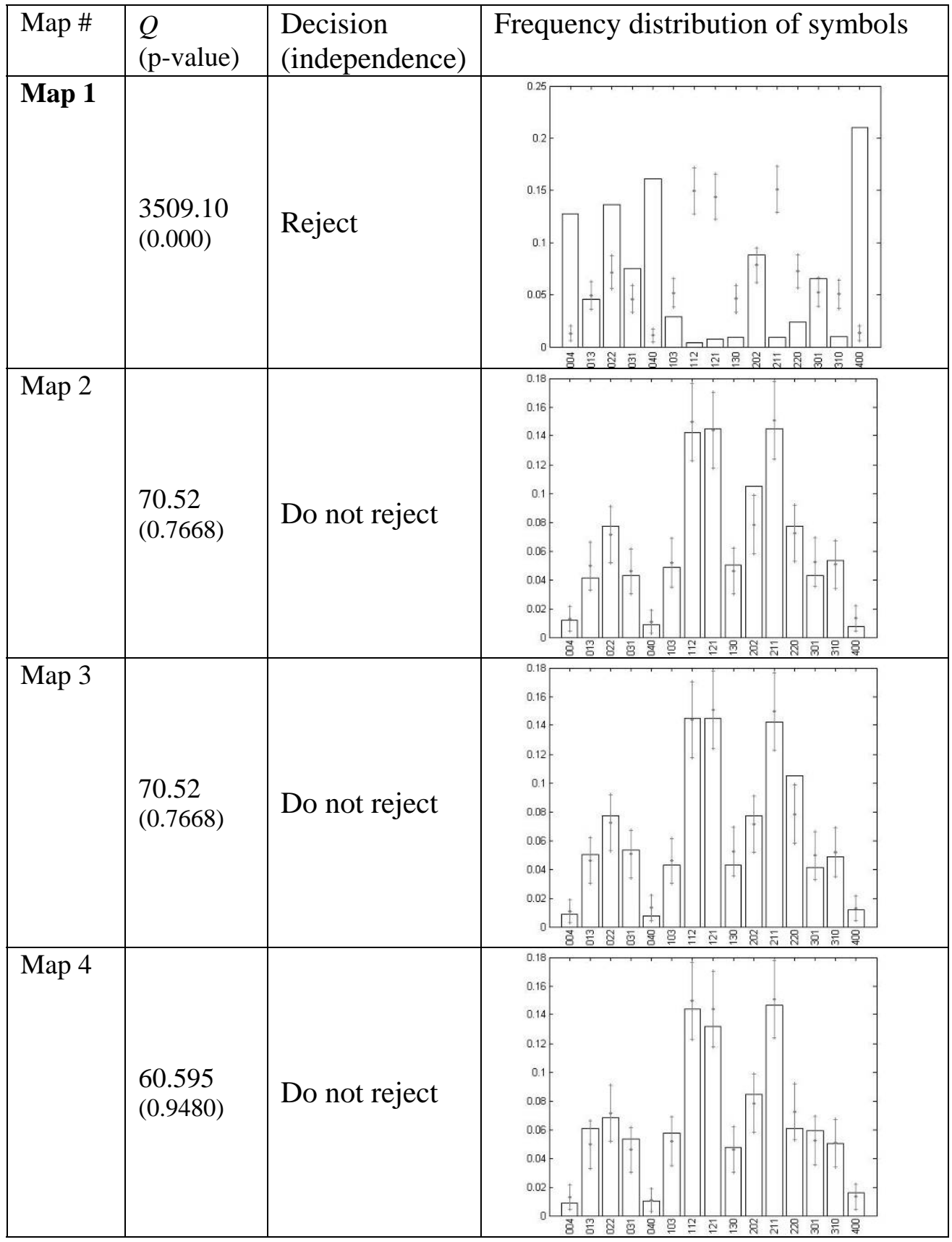

Table 2 (continued): $Q(m)$ test for spatial association (non-random maps in bold, $p$ values in parentheses). Test is conducted for $m=4$ and $r=1$. 


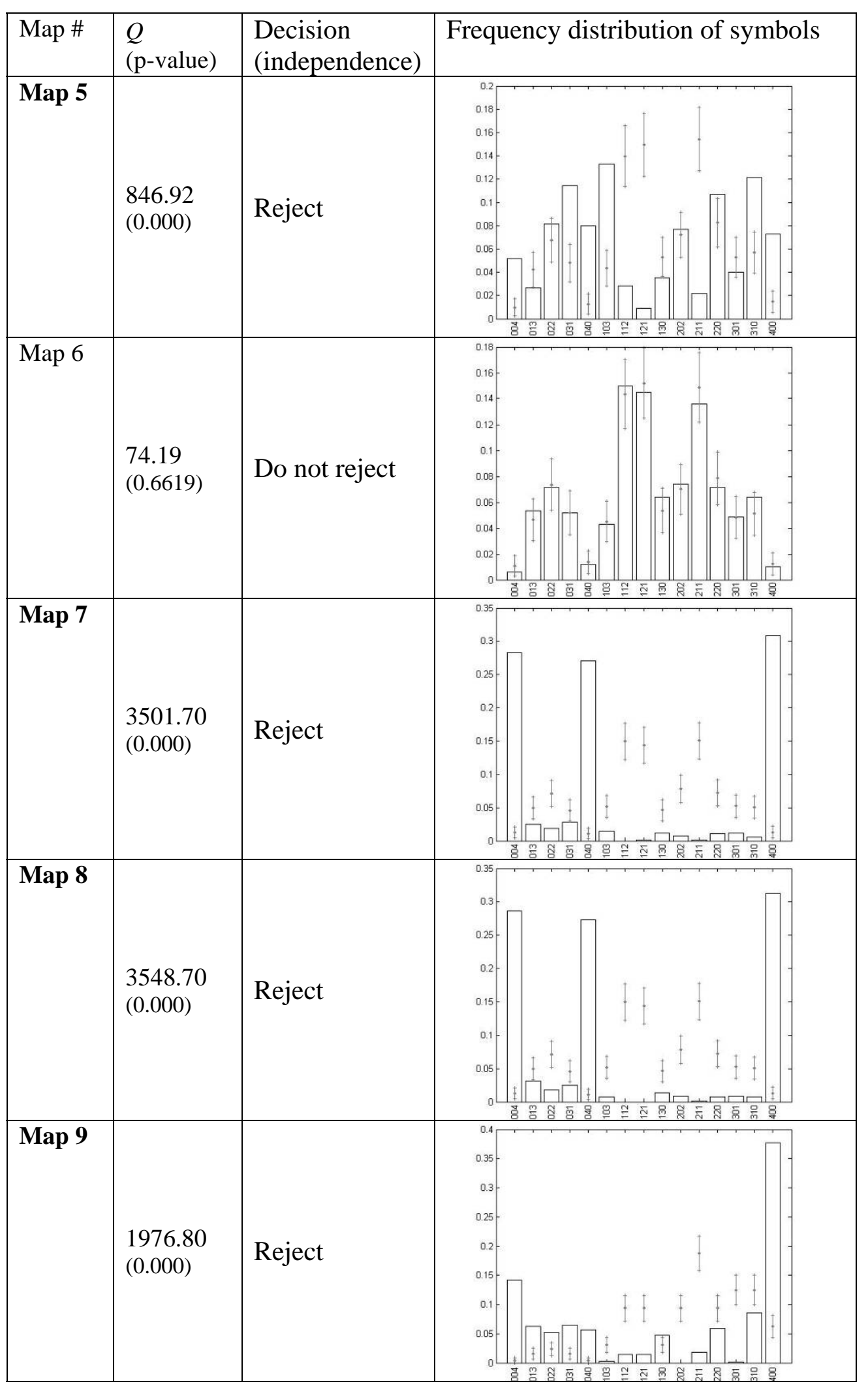

As seen in the table, the null hypothesis of spatial independence is rejected for Map [1], thus confirming the visual impression of a highly structured spatial distribution of variable values. As expected, the null hypothesis is rejected also for Maps [5], [7], and [8], since these maps (see Figure 2) were derived from Map [1] according to some 
organized principle (i.e., non-random transitions, rotation, and reflection, respectively). As well, the test also rejects the hypothesis of independence for Map [9], which was simulated using a strongly autocorrelated spatial process. The null hypothesis is not rejected for Maps [2], [3], and [4]. Likewise, the hypothesis is not rejected for Map [6], which was originally based on Map [1], but subjected to a process of random transitions.

At this point, our interest in the comparison of maps would practically be limited to pairs of maps that display a significant degree of spatial association. This would mean a pair-wise comparison of Maps [1] : [5] : [7] : [8] : [9]. For illustrative purposes, we calculate the statistics for all pairs of maps, as this serves to demonstrate the appropriateness of the statistics in a wide range of situations.

The next step in our comparison framework is to determine if two maps are compositionally equivalent, or in other words, to evaluate if the symbols appear with similar or significantly different frequency. The results of applying the $Q_{E}$ test to each pair of maps appear in Table 3 (calculation of the statistic is based on the same parameters used before, namely $m=4$ and $r=1$ ). First, note that a map is identical to itself, and therefore the statistic always fails to reject the null hypothesis of compositional equivalence when the two maps in the input are the same. When two maps are random, the expectation is that they will be equivalent in their composition, since by definition in a random map all symbols appear with similar frequencies (contingent on the frequency of classes; see frequency plots in Table 3 for Maps [2], [3], [4], and [6]). The test confirms this, by failing to reject the null hypothesis for the comparison of Maps [2] : [3] : [4] : [6]. Next, the expectation is that any spatially associated map will have a different composition when compared to any random map, which would lead to the conclusions that the two maps are different (and the comparison is relatively uninteresting, since one of the two maps is random). This intuition is again confirmed by the results in the table, where it can be seen that the null hypothesis of compositional equivalence is rejected for Maps [1], [5], [7], [8], and [9] : [2], [3], [4], and [6]. In other words, any two maps in these pairs are significantly different from each other.

The more interesting cases are when the two maps for the comparison display significant spatial association, or in other words, patterning. The results are again in full agreement with the setup of the example. First, we fail to reject the null hypothesis when comparing Maps [1] : [7] : [8]. This is logical, since the input maps in each case 
are essentially the same map, only with different orientations. In contrast, the null hypothesis is rejected when comparing Maps [1], [7], [8] : [5] : [9]. In the case of Maps [1], [7], and [8] : [5], this is to be expected since Maps [1], [7], and [8] have already been determined to be equivalent, and Map [5] was obtained from Map [1] using a transformation rule that changed its composition. As a result, Maps [5] is significantly different from the others. Finally, Map [9] was generated using a separate spatially structured process that was not constrained to generate the same composition as any of the preceding maps.

Table 3: $Q_{E}$ test for compositional equivalence (non-random maps in bold, and $p$-values in parentheses). Decision rule is to reject the hypothesis of equivalence. Shaded cells indicate reject.

Map 1 Map 2 Map 3 Map 4 Map 5 Map 6 Map 7 Map 8 Map 9

\begin{tabular}{|c|c|c|c|c|c|c|c|c|c|}
\hline Map 1 & $\begin{array}{c}0.0 \\
(1.000)\end{array}$ & $\begin{array}{l}1331.6 \\
(0.000)\end{array}$ & $\begin{array}{l}1335.4 \\
(0.000)\end{array}$ & $\begin{array}{l}1280.3 \\
(0.000)\end{array}$ & $\begin{array}{c}767.4 \\
(0.000)\end{array}$ & $\begin{array}{l}1318.3 \\
(0.000)\end{array}$ & $\begin{array}{c}63.4 \\
(0.914)\end{array}$ & $\begin{array}{c}53.8 \\
(0.989)\end{array}$ & $\begin{array}{c}362.6 \\
(0.000)\end{array}$ \\
\hline Map 2 & & $\begin{array}{c}0.0 \\
(1.000)\end{array}$ & $\begin{array}{c}61.9 \\
(0.933)\end{array}$ & $\begin{array}{c}73.9 \\
(0.670)\end{array}$ & $\begin{array}{c}463.1 \\
(0.000)\end{array}$ & $\begin{array}{c}66.6 \\
(0.858)\end{array}$ & $\begin{array}{l}1323.0 \\
(0.000)\end{array}$ & $\begin{array}{l}1338.7 \\
(0.000)\end{array}$ & $\begin{array}{c}922.4 \\
(0.000)\end{array}$ \\
\hline Map 3 & & & $\begin{array}{c}0.0 \\
(1.000)\end{array}$ & $\begin{array}{c}72.4 \\
(0.715)\end{array}$ & $\begin{array}{c}472.5 \\
(0.000)\end{array}$ & $\begin{array}{c}76.8 \\
(0.580)\end{array}$ & $\begin{array}{l}1308.9 \\
(0.000)\end{array}$ & $\begin{array}{l}1327.7 \\
(0.000)\end{array}$ & $\begin{array}{c}874.7 \\
(0.000)\end{array}$ \\
\hline Map 4 & & & & $\begin{array}{c}0.0 \\
(1.000) \\
\end{array}$ & $\begin{array}{c}441.8 \\
(0.000) \\
\end{array}$ & $\begin{array}{c}74.3 \\
(0.660) \\
\end{array}$ & $\begin{array}{l}1278.0 \\
(0.000) \\
\end{array}$ & $\begin{array}{l}1293.0 \\
(0.000) \\
\end{array}$ & $\begin{array}{c}871.4 \\
(0.000) \\
\end{array}$ \\
\hline Map 5 & & & & & $\begin{array}{c}0.0 \\
(1.000) \\
\end{array}$ & $\begin{array}{c}463.7 \\
(0.000)\end{array}$ & $\begin{array}{c}755.3 \\
(0.000) \\
\end{array}$ & $\begin{array}{c}780.1 \\
(0.000) \\
\end{array}$ & $\begin{array}{c}509.5 \\
(0.000) \\
\end{array}$ \\
\hline Map 6 & & & & & & $\begin{array}{c}0.0 \\
(1.000)\end{array}$ & $\begin{array}{l}1315.4 \\
(0.000)\end{array}$ & $\begin{array}{l}1327.6 \\
(0.000)\end{array}$ & $\begin{array}{c}875.7 \\
(0.000) \\
\end{array}$ \\
\hline Map 7 & & & & & & & $\begin{array}{c}0.0 \\
(1.000)\end{array}$ & $\begin{array}{c}40.0 \\
(1.000)\end{array}$ & $\begin{array}{c}359.6 \\
(0.000)\end{array}$ \\
\hline Map 8 & & & & & & & & $\begin{array}{c}0.0 \\
(1.000)\end{array}$ & $\begin{array}{c}343.6 \\
(0.000) \\
\end{array}$ \\
\hline Map 9 & & & & & & & & & $\begin{array}{c}0.0 \\
(1.000)\end{array}$ \\
\hline
\end{tabular}

The final step in our comparison framework is to test the hypothesis of independence between two maps. The objective is to determine whether two maps could be linked by a rule other than random transitions. As seen in Table 4, the $Q_{I}$ statistic (calculated with $m=1$ ) correctly rejects the null hypothesis when the two maps compared are the same: a map is not independent from itself.

We begin our discussion of these results with Map [1]. In this case, we observe that the null hypothesis is not rejected when the map used in the comparison is random (i.e. Maps [2], [3], [4], and [6]); as expected, an organized rule does not exist that could transform one map into the other, and the maps are independent. The null hypothesis is 
correctly rejected when comparing Maps [1]: [5], [7], [8]. Since an underlying rule exists that can be used to transform the maps (e.g., from [1] to [5] and vice versa if the rule is inverted), the maps are not independent. The test cannot identify the underlying rule, but can point to its existence.

With respect to Map [2], the statistic correctly rejects the hypothesis of independence when the comparison is made with respect to Map [3]. As seen in Figure 2, the two maps are random; however, Map [3] was derived from Map [2] using a set of systematic transitions rules. This result indicates that the statistic is able to detect patterns of dependency between maps, even if the two maps are random. When comparison is made to any of the other maps in the example, on the other hand, the test fails to reject the null hypothesis. This is precisely as expected, since the map is random and therefore independent from the other cases. The situation is the same for random maps [3], [4], and [6] when compared to any other maps (with the exception of the comparison between Maps [2] : [3] as per the preceding discussion).

Table 4: $Q_{I}$ test for independence (non-random maps in bold, and $p$-values in parentheses). Decision rule is to reject hypothesis of independence. Shaded cells indicate reject.

Map 1 Map 2 Map 3 Map 4 Map 5 Map 6 Map 7 Map 8 Map 9

\begin{tabular}{|c|c|c|c|c|c|c|c|c|c|}
\hline & Map 1 & Map 2 & Map 3 & Map 4 & Map 5 & Map 6 & Map 7 & Map 8 & Map 9 \\
\hline \multirow{2}{*}{ Map 1} & 4455.1 & 1.2 & 1.2 & 6.2 & 1942.3 & 1.1 & 1031.4 & 635.4 & 160.1 \\
\hline & $(0.000)$ & $(0.880)$ & $(0.880)$ & (0.183) & $(0.000)$ & $(0.893)$ & $(0.000)$ & $(0.000)$ & (0.000) \\
\hline \multirow{2}{*}{ Map 2} & & 4455.1 & 4455.1 & 2.5 & 3.0 & 1.3 & 6.7 & 3.0 & 2.6 \\
\hline & & $(0.000)$ & $(0.000)$ & (0.642) & $(0.559)$ & $(0.861)$ & $(0.152)$ & $(0.560)$ & (0.629) \\
\hline \multirow{2}{*}{ Map 3} & & & 4455.1 & 2.5 & 3.0 & 1.3 & 6.7 & 3.0 & 2.6 \\
\hline & & & $(0.000)$ & $(0.642)$ & $(0.559)$ & $(0.861)$ & $(0.152)$ & $(0.560)$ & $(0.629)$ \\
\hline \multirow{2}{*}{ Map 4} & & & & 4455.1 & 2.0 & 4.1 & 2.5 & 2.5 & 2.3 \\
\hline & & & & $(0.000)$ & $(0.732)$ & $(0.389)$ & $(0.637)$ & $(0.645)$ & $(0.687)$ \\
\hline \multirow{2}{*}{ Map 5} & & & & & 4452.5 & 7.0 & 282.8 & 258.1 & 46.8 \\
\hline & & & & & $(0.000)$ & $(0.134)$ & $(0.000)$ & $(0.000)$ & $(0.000)$ \\
\hline \multirow{2}{*}{ Map 6} & & & & & & 4454.9 & 2.0 & 3.1 & 0.9 \\
\hline & & & & & & $(0.000)$ & $(0.744)$ & $(0.534)$ & $(0.921)$ \\
\hline \multirow{2}{*}{ Map 7} & & & & & & & 4455.1 & 321.9 & 239.5 \\
\hline & & & & & & & $(0.000)$ & $(0.000)$ & $(0.000)$ \\
\hline \multirow{2}{*}{ Map 8} & & & & & & & & 4455.1 & 258.0 \\
\hline & & & & & & & & $(0.000)$ & $(0.000)$ \\
\hline \multirow{2}{*}{ Map 9} & & & & & & & & & 4217.1 \\
\hline & & & & & & & & & $(0.000)$ \\
\hline
\end{tabular}

Application of the test to Maps [5] : [7] : [8] leads to rejection of the null hypothesis, for the evident reason that underlying transition rules exist among these maps through their connection with Map [1]. 
An intriguing case is Map [9]. The statistic rejects the hypothesis of independence when the comparison is made with respect to Maps [1], [5], [7], and [8]. As previously explained, this map was generated using a separate spatial autocorrelation process, and Map [9] is therefore not directly derived from any of these maps. Considering the high degree of spatial association in the map, on the other hand, it is perfectly plausible that some set of non-random transformations could exist to link Maps [1], [5], [7], [8] : [9]. These results offer a cautionary counter-example in the application of the statistic: quite simply, lack of independence does not necessarily imply causality. This point should be clear to anyone familiar with elementary statistics and the basic tenet that correlation is not causation. While the statistic appears to do a reasonable job of identifying lack of independence due to causal processes, the temptation should be avoided to infer causality based on this test.

\section{Conclusions}

In this paper we have introduced a new approach for the comparison of thematic maps. Our approach sequentially tries to identify differences between maps by assessing the degree of spatial association of each map and the compositional equivalence between maps based on map segments (as opposed to map cells/elements), and by testing for independence between the two maps. The framework covers a wide range of cases of practical interest, and provides a complete inferential framework with clearly defined null hypotheses at each step of the sequence. While the issue of significance testing in map comparison appears to be somewhat controversial, we would argue that testing for well defined hypotheses, such as introduced in this paper, can enhance exploratory analysis by clarifying the relationships between maps.

In order to implement our map comparison framework, we have introduced two new statistics for the spatial analysis of qualitative data. The statistics are essential to our approach, but will likely be of interest in a broader variety of settings: for instance, $Q_{E}$ can be applied to maps in different regions and/or with different zoning systems to assess similarities in composition. One limitation of our approach is that maps can be tested for differences at a given level of confidence. However, unlike the method of Wulder et al. (2004) that can detect clusters of differences between maps, when differences are detected in our framework, the statistics do not directly indicate where they happen. Therefore, our approach is still lacking tools for the identification of clusters of coincidences or differences. This is a matter for future research. 
Also, in terms of additional research, in this paper we introduced two new statistics, that were shown to perform well in the example. The finite sample properties of $Q(m)$ were already investigated in Ruiz et al. (2010). The next step is to investigate, using carefully designed simulation experiments, the small sample properties of $Q_{E}(m)$ and $Q_{I}$, in order to ascertain their size and power under different sample sizes, size of $m$-surrounding, degree of overlap $r$, and level of spatial association in each map. This is the subject of ongoing research.

\section{References}

Boots B, Csillag F (2006) Categorical maps, comparisons, and confidence. Journal of Geographical Systems 8(2):109-118

Cliff AD, Ord JK (1981) Spatial Processes: Models and Applications. Pion, London

Congalton RG (1988) Using Spatial Auto-Correlation Analysis to Explore the Errors in Maps Generated from Remotely Sensed Data. Photogrammetric Engineering and Remote Sensing 54(5):587-592

Dacey MF (1968) A Review on Measures of Contiguity for Two and k-Color Maps. In: Berry, B. J. L. and MArble, D. F. (ed) Spatial Analysis: A Reader in Statistical Geography. Prentice Hall, Englewood Cliffs, NJ, 479-495

Foody GM (2002) Status of land cover classification accuracy assessment. Remote Sensing of Environment 80(1):185-201

Foody GM (2004) Thematic map comparison: Evaluating the statistical significance of differences in classification accuracy. Photogrammetric Engineering and Remote Sensing 70(5):627-633

Fotheringham AS, Brunsdon C (1999) Local forms of spatial analysis. Geographical Analysis 31(4):340-358

Gustafson EJ (1998) Quantifying landscape spatial pattern: What is the state of the art? Ecosystems 1(2):143-156

Hagen A (2003) Fuzzy set approach to assessing similarity of categorical maps. International Journal of Geographical Information Science 17(3):235-249

Hagen-Zanker A (2006) Map comparison methods that simultaneously address overlap and structure. Journal of Geographical Systems 8(2):165-185

Hagen-Zanker A (2009) An improved Fuzzy Kappa statistic that accounts for spatial autocorrelation. International Journal of Geographical Information Science 23(1):61-73

Hagen-Zanker A, Straatman B, Uljee I (2005) Further developments of a fuzzy set map comparison approach. International Journal of Geographical Information Science 19(7):769-785 
Kuhnert M, Voinov A, Seppelt R (2005) Comparing raster map comparison algorithms for spatial modeling and analysis. Photogrammetric Engineering and Remote Sensing 71(8):975-984

Lehmann EL (1986) Testing statistical hypotheses. John Wiley, New York

Mas JF (1999) Monitoring land-cover changes: a comparison of change detection techniques. International Journal of Remote Sensing 20(1):139-152

Pontius RG, Shusas E, McEachern M (2004) Detecting important categorical land changes while accounting for persistence. Agriculture Ecosystems \& Environment 101(2-3):251-268

Remmel TK (2009) Investigating Global and Local Categorical Map Configuration Comparisons Based on Coincidence Matrices. Geographical Analysis 41(2):144-157

Rohatgi VK (1976) An introduction to probability theory and mathematical statistics. Wiley, New York

Ruiz M, Lopez F, Páez A (2010) Testing for spatial association of qualitative data using symbolic dynamics. Journal of Geographical Systems (in press; doi: 10.1007/s10109009-0100-1)

Soon SYT (1996) Binomial Approximation for Dependent Indicators. Statistica Sinica 6703-714

Stehman SV (1999) Comparing thematic maps based on map value. International Journal of Remote Sensing 20(12):2347-2366

Wulder MA, Boots B, Seemann D, White JC (2004) Map comparison using spatial autocorrelation: an example using AVHRR derived land cover of Canada. Canadian Journal of Remote Sensing 30(4):573-592 